\title{
Editorial
}

\section{Black Holes Physics}

\author{
Xiaoxiong Zeng, ${ }^{1}$ Christian Corda, ${ }^{2,3,4}$ and Deyou Chen ${ }^{5}$ \\ ${ }^{1}$ School of Science, Chongqing Jiaotong University, Nanan 400074, China \\ ${ }^{2}$ Dipartimento di Fisica e Chimica, Istituto Universitario di Ricerca Scientifica "Santa Rita," Centro di Scienze Naturali, \\ Via di Galceti, 74, 59100 Prato, Italy \\ ${ }^{3}$ Institute for Theoretical Physics and Advanced Mathematics (IFM) Einstein-Galilei, Via Santa Gonda 14, 59100 Prato, Italy \\ ${ }^{4}$ International Institute for Applicable Mathematics \& Information Sciences (IIAMIS), B.M. Birla Science Centre, \\ Adarsh Nagar, Hyderabad 500 463, India \\ ${ }^{5}$ Institute of Theoretical Physics, China West Normal University, Nanchong, Sichuan 637009, China
}

Correspondence should be addressed to Xiaoxiong Zeng; xxzeng@mail.bnu.edu.cn

Received 15 May 2014; Accepted 15 May 2014; Published 28 May 2014

Copyright (C) 2014 Xiaoxiong Zeng et al. This is an open access article distributed under the Creative Commons Attribution License, which permits unrestricted use, distribution, and reproduction in any medium, provided the original work is properly cited. The publication of this article was funded by $\mathrm{SCOAP}^{3}$.

Black hole $(\mathrm{BH})$ is perhaps the most fascinating object in the research fields of astrophysics and gravitational physics. This mysterious object is predicted by the general theory of relativity (GTR), but such a classical theory cannot explain all its properties. It is believed that only a more general, definitive theory of quantum gravity, which should unify GTR with quantum mechanics, should clarify all the mysteries of $\mathrm{BH}$ physics, starting from the unsolved problem of the singularity in the BH's core to arrive to the $\mathrm{BH}$ information puzzle and to the last stages of the $\mathrm{BH}$ evaporation, where very high energies are involved. In fact, it is general conviction that black holes result in highly excited states representing both the "hydrogen atom" and the "quasi-thermal emission" in quantum gravity. On this issue we recall that an exciting consequence of $\mathrm{TeV}$-scale quantum gravity could be the potential production of mini-BHs in high-energy experiments, like the LHC and beyond.

This special issue on $\mathrm{BH}$ physics consists of 17 interesting and well written papers.

The paper "A little quantum help for cosmic censorship and a step beyond all that", by N. Pappas, discusses the weak and strong versions of the cosmic censorship conjecture and also deals with the well-known problem of naked singularities.

The paper "On the critical phenomena and Thermodynamics of the Reissner-Nordstrom-de Sitter black hole," by R. Zhao et al., deals with the effective thermodynamic quantities in Reissner-Nordstrom-de Sitter $\mathrm{BH}$ by also discussing its thermodynamic stability.
The paper "Intermediate mass black holes: their motion and associated energetics," by C. Sivaram and A. Kenath, is devoted to exploring the astrophysical signatures and evidences for the intermediate mass BHs. Especially authors describe the specific features of their motion and energetics, related with the Bondi accretion.

The paper "Energy Loss of a heavy particle near $3 d$ rotating hairy black hole," by J. Naji and H. Saadat, considers rotating $\mathrm{BH}$ in 3 dimensions with a scalar charge and discusses energy loss of heavy particle moving near the $\mathrm{BH}$ horizon.

The paper "Holographic screens in ultraviolet self-complete quantum gravity," by P. Nicolini and E. Spallucci, investigates the idea of a short distance fundamental scale below which it is not possible to probe and analyzes the geometry and thermodynamics of a holographic screen in the framework of the ultraviolet self-complete quantum gravity.

The paper "Black holes and quantum mechanics," by B. G. Sidharth, reconsiders BHs in the context of general relativity critically reviewing all problems relating these phenomena.

The paper "Magnetic string with a nonlinear U(1) source," by Seyed H. Hendi, deals with the study of magnetic string solutions in Einstein gravity in the presence of nonlinear electrodynamics. Also the effects of these nonlinear fields as well as other properties of the solutions are investigated, precisely.

The paper "Researching on Hawking effect in a Kerr space time via open quantum system approach," by X.-M. Liu and W.-B. Liu, investigates the Hawking effect in a Kerr space time 
in the framework of open quantum systems, showing that Hawking effect of the Kerr space time can also be understood as the manifestation of thermalization phenomena via open quantum system approach.

The paper "Holograghic Brownian motion in three dimensional Gödel black hole," by J. Sadeghi et al., uses the AdS/CFT correspondence and Gödel $\mathrm{BH}$ background to study the dynamics of heavy quark under a rotating plasma.

The paper "Entropy spectrum of a KS black hole in IR modified Hovrava-Lifshitz gravity," by S. Zhou et al., discusses the entropy spectrum and area spectrum of a KS BH based on the proposal of adiabatic invariant quantity. It is found that that the entropy spectrum is discrete and equidistant spaced and the area spectrum is not equidistant spaced, which depends on the parameter of gravity theory.

The paper "Particle collisions in the lower dimensional rotating black hole space-time with the cosmological constant," by J. Yang et al., deals with the effect of ultrahigh energy collisions of two particles with different energies near the horizon of a $2+1$ dimensional BTZ BH (BSW effect), finding that the particle with the critical angular momentum could exist inside the outer horizon of the BTZ BH regardless of the particle energy.

The paper "The geometry of black hole singularities," by O. C. Stoica, is a review about singularity problem in general and $\mathrm{BH}$ singularity in particular. The importance of dimensional reduction effects is also stressed.

The paper "State-space geometry, statistical fluctuations and black holes in string theory," by S. Bellucci and B. N. Tiwari, considers statistical properties of the charged and anticharged $\mathrm{BH}$ configurations by using the notion of the thermodynamic geometry. The authors highlight the utility of thermodynamic geometry in understanding the state space correlations and fluctuations in BHs in string theory while treating them thermodynamically.

The paper "Quantum tunnelling for Hawking radiation from both static and dynamic black hole," by S. Chakraborty and S. Saha, deals with the well-known Hawking radiation and quantum corrections in order to further improve the theory from the semiclassical approach. The authors specially study the Hawking radiation from both static and nonstatic spherically symmetric BHs.

The paper "Electrostatics in the surroundings of a topologically charged black hole in the brane," by A. Larrañaga et al., studies the EM properties of $4 \mathrm{D} \mathrm{BH}$ due to brane contributions, determining the electrostatic potential generated by a static point-like charge in the brane-world space-time of a $\mathrm{BH}$ with topological (or tidal) charge.

The paper "Analyzing black hole super-radiance emission of particles/energy from a black hole as a Gedankenexperiment to get bounds on the mass of a graviton," by A. Beckwith, discusses the process of particles emission and adopted a standard approach proposed by Padmanabhan. In the author's point of view "super-radiance allows massive gravity to be consistent with $\mathrm{BH}$ physics and general relativity."

The paper "Hawking radiation-quasi-normal modes correspondence and effective states for non-extremal ReissnerNordstrom black holes," by C. Corda et al., investigates the correspondence between Hawking radiation and $\mathrm{BH}$ quasi-normal modes and defines the concept of "effective state" for the non-extremal Reissner-Nordstrom black holes. The work is the extension of earlier works of the same research group and contributes to the understanding of the quantum properties of BHs.

Xiaoxiong Zeng Christian Corda Deyou Chen 

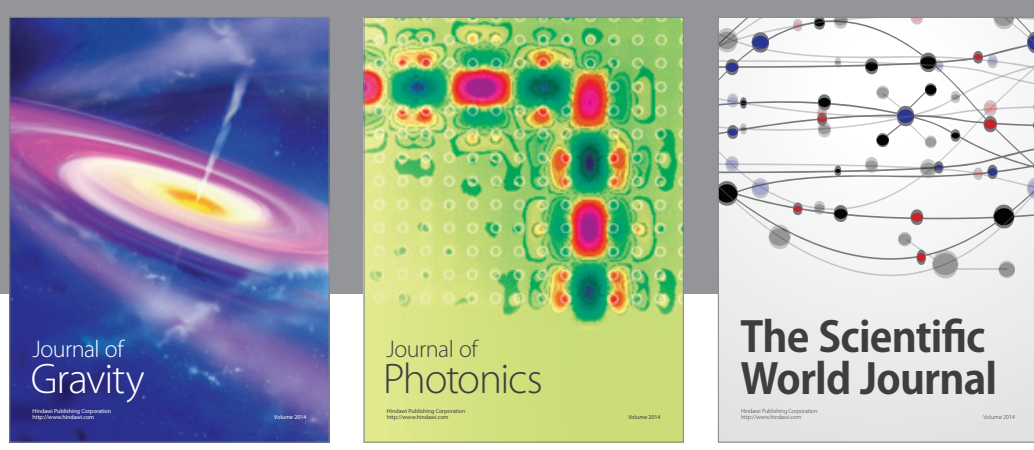

The Scientific World Journal
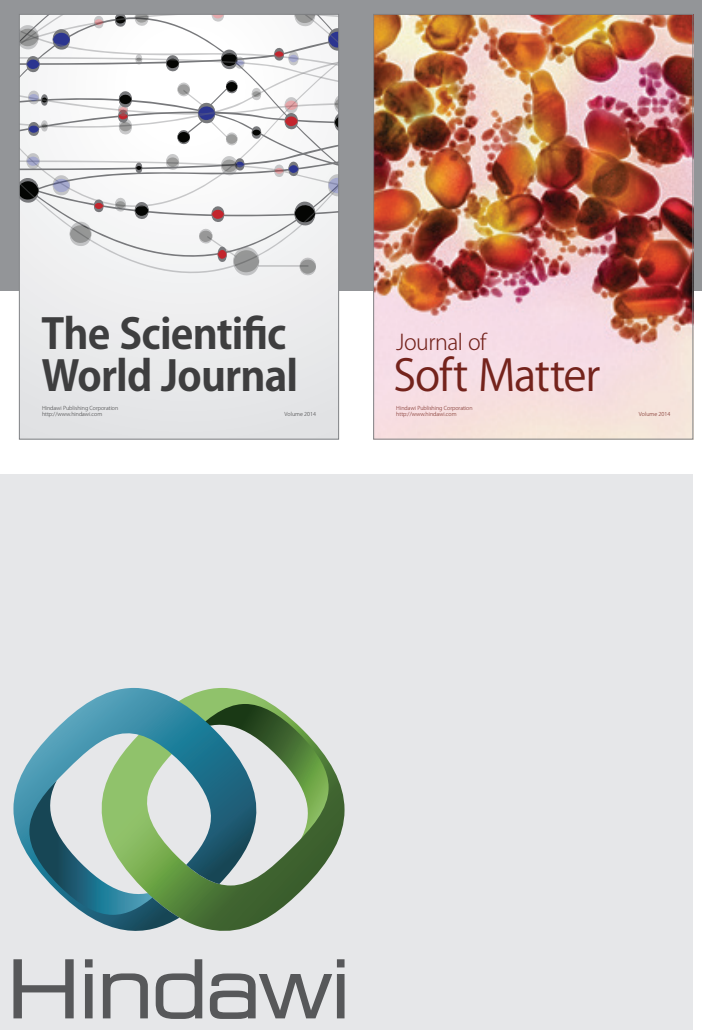

Submit your manuscripts at

http://www.hindawi.com

nternational Journal of

Statistical Mechanics
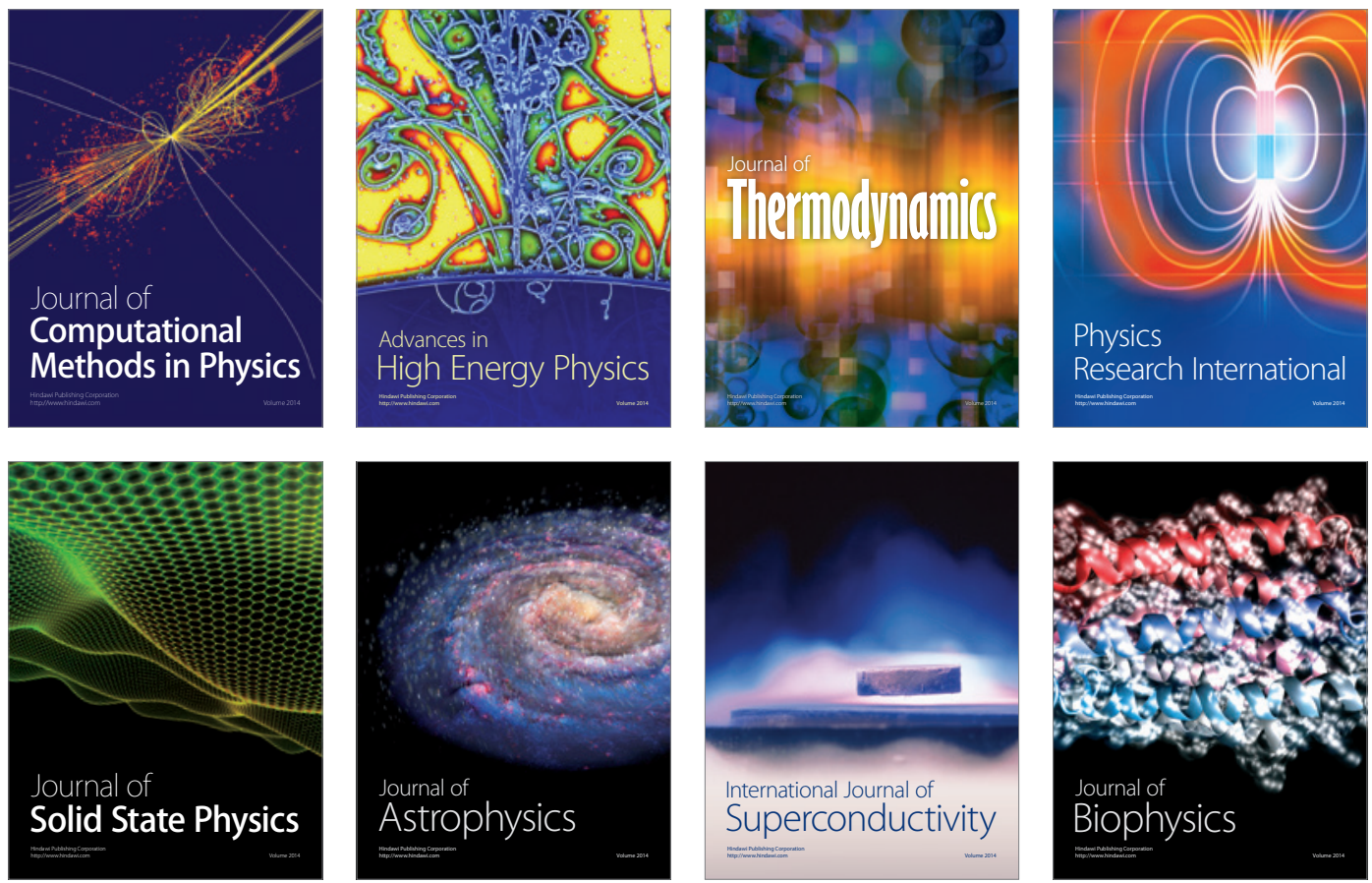
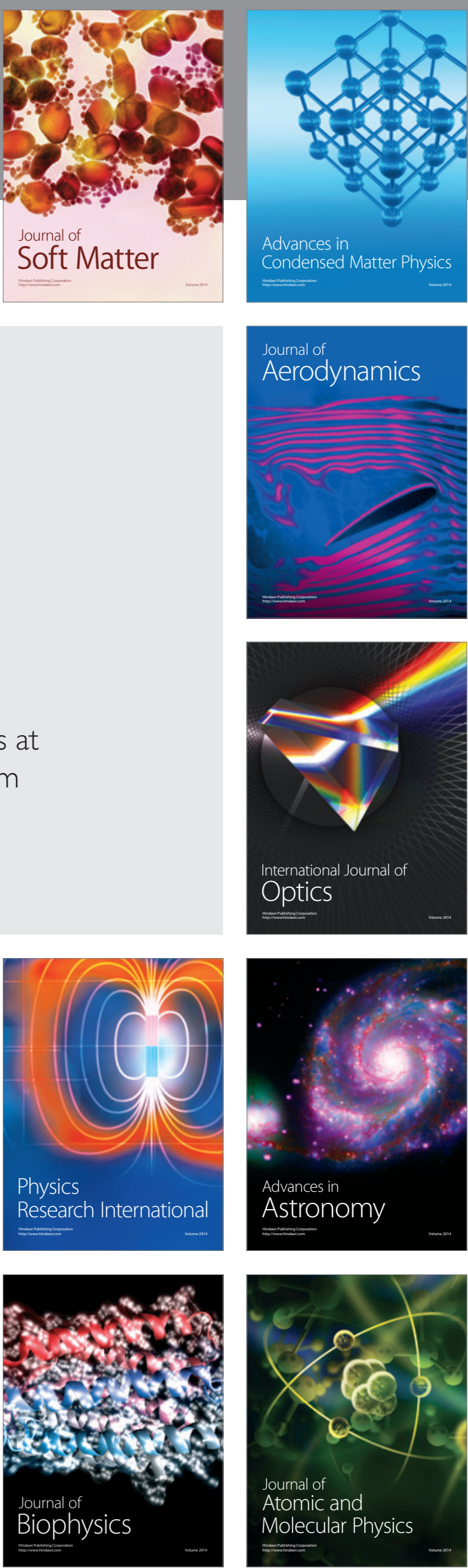